\title{
RESEARCH
}

Open Access

\section{Adjuvant tegafur-uracil (UFT) or S-1 monotherapy for advanced gastric cancer: a single center experience}

\author{
Hung-Hsuan Yen ${ }^{1,2}$, Chiung-Nien Chen², Chi-Chuan Yeh ${ }^{2,3}$ and I-Rue Lai ${ }^{2,4^{*}}$
}

\begin{abstract}
Background: Adjuvant tegafur-gimeracil-oteracil (S-1) is commonly used for gastric cancer in Asia, and tegafururacil (UFT) is another oral fluoropyrimidine when $\mathrm{S}-1$ is unavailable. The real-world data of adjuvant UFT has less been investigated.
\end{abstract}

Methods: Patients with pathological stage II-IIIB (except T1) gastric cancer receiving adjuvant UFT or S-1 monotherapy after D2 gastrectomy were included. Usage of UFT or S-1 was based on reimbursement policy of the Taiwanese healthcare system. The characteristics, chemotherapy completion rates, and 5-year recurrence-free survival (RFS) and overall survival (OS), were compared between these two groups.

Results: From 2005 to 2016, 86 eligible patients were included. Most tumor characteristics were similar between the UFT group ( $n=37$; age $59.1 \pm 13.9$ years) and S-1 group ( $n=49$; age $56.3 \pm 10.7$ years), except there were significantly more Borrmann type III/IV (86.5\% versus 67.3\%; $p=0.047)$ and T4 (56.8\% versus $10.2 \% ; p<0.001)$ lesions in the UFT group than in the S-1 group. The chemotherapy complete rates were similar in the two groups. The 5 -year RFS was $56.1 \%$ in the UFT group and $59.6 \%$ in the S-1 group $(p=0.71)$, and the 5 -year OS was $78.3 \%$ in the UFT group and $73.1 \%$ in the S-1 group $(p=0.48)$. The hazard ratio of adjuvant chemotherapy (S-1 versus UFT) on RFS was 1.25 (95\% confidence interval $=0.53-2.94$ ) when Borrmann type and $\mathrm{T}$ and $\mathrm{N}$ stages were adjusted.

Conclusions: This small cohort study showed adjuvant UFT, and S-1 monotherapy had a comparable long-term outcome for pathological stage II-IIIB gastric cancer following D2 gastrectomy.

Keywords: Adjuvant chemotherapy, Gastric cancer, S-1, Tegafur, UFT

\footnotetext{
*Correspondence: iruelai@gmail.com

2Department of Surgery, National Taiwan University Hospital, No. 7 Chung-Shan South Rd, Zhongzheng District, Taipei 10002, Taiwan

${ }^{4}$ Graduate Institute of Anatomy and Cell Biology, College of Medicine, National Taiwan University, Taipei, Taiwan

Full list of author information is available at the end of the article
}

(C) The Author(s). 2021 Open Access This article is licensed under a Creative Commons Attribution 4.0 International License, which permits use, sharing, adaptation, distribution and reproduction in any medium or format, as long as you give appropriate credit to the original author(s) and the source, provide a link to the Creative Commons licence, and indicate if changes were made. The images or other third party material in this article are included in the article's Creative Commons licence, unless indicated otherwise in a credit line to the material. If material is not included in the article's Creative Commons licence and your intended use is not permitted by statutory regulation or exceeds the permitted use, you will need to obtain permission directly from the copyright holder. To view a copy of this licence, visit http://creativecommons.org/licenses/by/4.0/ The Creative Commons Public Domain Dedication waiver (http://creativecommons.org/publicdomain/zero/1.0/) applies to the data made available in this article, unless otherwise stated in a credit line to the data. 


\section{Background}

Gastric cancer has been the 3rd most deadly cancer worldwide [1]. Radical gastrectomy with lymph node dissection is the treatment of choice for resectable gastric cancer [2], and adjuvant chemotherapy is recommended for pathological stage II-IIIB gastric cancer based on several randomized controlled trials (RCTs), Japanese gastric cancer treatment guidelines, and Korean practice guideline for gastric cancer [3-12].

Dihydropyrimidine dehydrogenase (DPD) inhibitory fluoropyrimidine (DIF), the oral form prodrug of 5flurouracil (5-FU), has been developed since 1980 [10], and is currently the most important chemotherapeutic agent used in adjuvant chemotherapy for advanced gastric cancer. Tegafur-uracil (UFT) is the first generation of oral DPD DIF, followed by tegafur-gimeracil-oteracil (S-1), the next generation of oral DPD DIF. S-1 contains oteracil that inhibits phosphorylation of 5 -FU within the gastrointestinal mucosal cells and thus theoretically reduces the gastrointestinal toxicity [13].

UFT was firstly used as an adjuvant monotherapy for pathological T2N1-2 gastric cancer in 1997 [9], but the introduction of S-1 has replaced UFT gradually since 2001. In addition, S1 monotherapy has further become the standard adjuvant therapy for advanced gastric cancer in 2007 since the results of Adjuvant Chemotherapy Trial of TS-1 for Gastric Cancer (ACTS-GC) was published [7, 8, 14]. In Taiwan, UFT was approved for advanced gastric cancer and reimbursed by the Taiwanese healthcare system in October 2000; on the other hand, S-1 was approved for advanced gastric cancer based on the same inclusion criteria in the ACTS-GC in April 2010 but was not reimbursed until December 2016. Although both UFT and S-1 are indicated for advanced gastric cancer as adjuvant monotherapy in Taiwan, realworld experiences and comparisons of efficacy and tolerance between these two oral DPD DIFs remain suboptimal and are only limited to sub-group analysis in one clinical trial, the Stomach cancer Adjuvant Multi-Institutional group Trial (SAMIT) [15].

In the era of coronavirus disease (COVID-19) pandemic, the supply chain of chemotherapeutic agents could be no longer guaranteed. Re-evaluation of the existing alternative in the adjuvant treatment may be necessary. In this study, we shared our experiences of using UFT or S-1 monotherapy as adjuvant treatment for pathological stage II-IIIB gastric cancer after D2 gastrectomy, based on the unique historical cohorts formed by the different timings when UFT and S-1 were available in Taiwan.

\section{Methods}

\section{Study design and identification of the study cohort}

This is a retrospective cohort study for patients receiving adjuvant UFT or S-1 monotherapy after D2 gastrectomy by two senior surgeons (I.-R. L. and C.-N. C) in a tertiary referral medical center from 2005 to 2016. Patients with pathological stage II-IIIB gastric adenocarcinoma (excluding T1 cases), based on the American Joint Committee on Cancer (AJCC) 8th staging system, were included in the study. The surgical procedure followed the principles provided by Japanese Gastric Cancer Association $[11,16,17]$. The patients, who, declined adjuvant chemotherapy, had a positive margin on final pathology report, were treated with neoadjuvant chemotherapy, had concomitant malignancy, were lost to follow-up (no-show since the first postoperative outpatient followup), had operative mortality (death occurring $<1$ month of the index gastrectomy), or received adjuvant chemotherapy other than UFT or S-1, were excluded from the analysis. For patients who were lost to follow-up or had operative mortality, discussion and decision of the adjuvant chemotherapy were not made. A total of 262 patients had been screened initially and 86 patients who met the criteria were included (Fig. 1). The study was approved by the Institutional Review Board of National Taiwan University Hospital.

\section{Treatment and follow-up}

All the included patients were evaluated for and began adjuvant chemotherapy within 6 weeks after surgery. The eligible patients were classified into two groups, the UFT and S-1 groups, based on the type of adjuvant chemotherapy. During the period between 2005 and 2010, UFT was the only available oral fluoropyrimidine for advanced gastric cancer as adjuvant chemotherapy. Then S-1, the next generation of oral fluoropyrimidine, was introduced to our hospital since 2010. The UFT group was given oral UFT $267 \mathrm{mg} / \mathrm{m}^{2}$ in two or three doses per day for 28 days a course for 24 course $(96$ weeks) [15], and the S-1 group was given oral S-1 80 $\mathrm{mg} / \mathrm{m}^{2}$ in two doses per day for 28 days followed by 14 days rest for 8 courses (48 weeks) $[7,8]$. The dosage of UFT or S-1 was reduced if any intolerable adverse event or $\geq$ grade 3 adverse event occurred. Although we used the same ACTS-GC protocol in the S-1 group, we always extended the total number of chemotherapy course from 8 courses (48 weeks) to 12 courses (72 weeks) if dose de-escalation was required [18, 19]. The general principles for discontinuing UFT or S-1 in our routine practice included the following: (1) recurrence or death; (2) adverse events, more than 29 days of unresolved events that prevented starting or continuing a course; and (3) patients' preference. Completion of the adjuvant chemotherapy was defined as patients who had finished 24 courses for UFT or 8 courses for S-1 regardless of any dose reduction or schedule modification.

All patients received outpatient follow-up every 3 months during the first 2 years and then every 6 months 


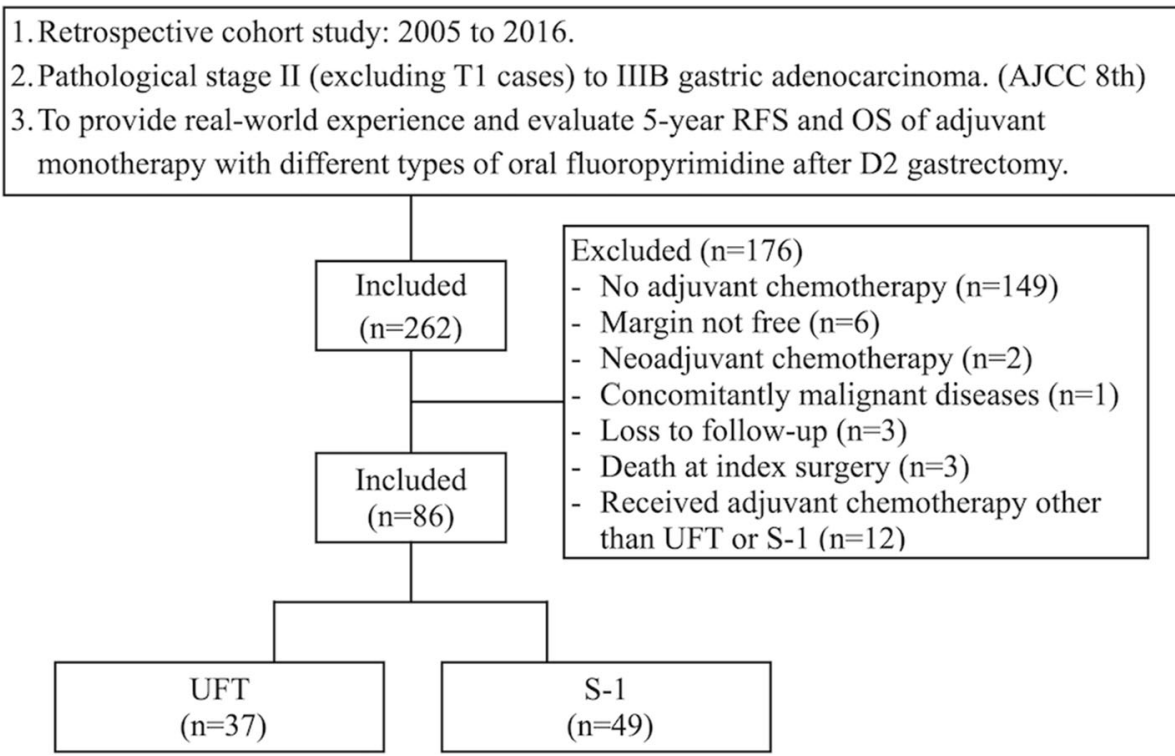

Fig. 1 Flow diagram of the subjects for the study. AJCC, American Joint Cancer Committee; RFS, recurrence-free survival; OS, overall survival; UFT, tegafur-uracil; S-1, tegafur-gimeracil-oteracil

from the third to fifth year postoperatively. Weight recording, symptom inquiry, and physical examination, were conducted at every outpatient follow-up. Blood sample for routine complete blood count, basic chemistry panel (liver and renal functions), and tumor marker (carcinoembryonic antigen) was collected every time before follow-up. Esophagogastroduodenoscopy was routinely performed at 1 year after surgery, and computed tomography scan of the abdomen and pelvis was performed every 6 months until the fifth year after surgery. The schedule of computed tomography scan, esophagogastroduodenoscopy or outpatient visit would be brought forward or increased in frequency if patients exhibited signs suspicious of recurrence. All these follow-up data were kept in either paper-based or electronic medical record.

The recurrence-free survival (RFS) was defined as the time between chemotherapy start date and the first event (all-cause death, recurrence of gastric cancer, or occurrence of a second cancer), and the overall survival (OS) was defined as the time between chemotherapy start date and all-cause death. The long-term follow-up data were acquired from the electronic medical record and database maintained by Cancer Registry, Cancer Administration and Coordination Center in our hospital. Patients lost to follow-up were censored. All the adverse events were reported based on the Common Terminology Criteria for Adverse Events, version 4.03.

\section{Statistical analysis}

Basic characteristics, including demographic data, operative methods, tumor classifications [20], pathological features and staging [16], and follow-up duration, were recorded and compared between the UFT and S- 1 groups. The adjuvant chemotherapy completion rates, sites of the first recurrence, and adverse events were also compared between these two groups. The chi-squared test or Fisher's exact test was used for qualitative variables as appropriate, and the Student's $t$ test was used for quantitative variables. We assessed time-to-event endpoint with the Kaplan-Meier method, and estimated the hazard ratio (HR) and its twosided 95\% confidence interval (CI) with the Cox regression model. Multivariate analysis for RFS was performed using Cox proportional hazards model; risk factors significant $(p<0.05)$ in the univariate analysis (Borrmann type) or considered important (type of chemotherapy, $\mathrm{T}$, and $\mathrm{N}$ stages) were included. All statistical tests were two-sided, and a $p$ value $<0.05$ denoted statistical significance. All statistical analyses were performed using $\mathrm{R}$ version 3.4.0.

\section{Results}

From 2005 to 2016, totally 86 gastric cancer patients with pathological stage II-IIIB (AJCC 8th edition) receiving adjuvant UFT or S-1 monotherapy after D2 gastrectomy by two senior surgeons (I.-R. L. and C.-N. C) were included in the study (Fig. 1). The UFT and S-1 groups comprised 37 and 49 patients, respectively, and there was no crossover between these two groups (Fig. 1).

Table 1 summarizes the basic characteristics of the UFT and S-1 groups. There was no statistical difference in most variables, including sex ratio, age, operative method, and most tumor characteristics, except for the Bormann type, $\mathrm{T}$ stage, and follow-up duration. More Borrmann type III/IV (86.5\% versus $67.3 \%$; $p=0.047$ ) 
Table 1 Basic characteristics between patients receiving UFT or S-1 as adjuvant chemotherapy

\begin{tabular}{|c|c|c|c|c|c|c|c|}
\hline & All & $(n=86)$ & UFT & $(n=37)$ & S-1 & $(n=49)$ & $p$ value \\
\hline$\overline{\text { Sex (male) }}$ & 56 & $(65.1)$ & 25 & $(67.6)$ & 31 & $(63.3)$ & 0.85 \\
\hline Age (years old) & 57.5 & \pm 12.2 & 59.1 & \pm 13.9 & 56.3 & \pm 10.7 & 0.31 \\
\hline Operative method & & & & & & & 0.10 \\
\hline Distal gastrectomy & 65 & $(75.6)$ & 26 & $(70.3)$ & 39 & $(79.6)$ & \\
\hline Total gastrectomy & 18 & $(20.9)$ & 11 & $(29.7)$ & 7 & $(14.3)$ & \\
\hline Proximal gastrectomy & 3 & $(3.5)$ & 0 & (0) & 3 & $(6.1)$ & \\
\hline Site & & & & & & & 0.33 \\
\hline Antrum and low body & 61 & $(70.9)$ & 26 & $(70.3)$ & 35 & $(71.4)$ & \\
\hline Middle and high body & 18 & $(20.9)$ & 9 & $(24.3)$ & 9 & $(18.4)$ & \\
\hline Whole stomach & 1 & $(1.2)$ & 1 & $(2.7)$ & 0 & (0) & \\
\hline Remnant & 6 & $(7.0)$ & 1 & $(2.7)$ & 5 & $(10.2)$ & \\
\hline Size $(\mathrm{cm})$ & 4.4 & \pm 2.0 & 4.3 & \pm 2.0 & 4.6 & \pm 2.1 & 0.49 \\
\hline Bormann type & & & & & & & $0.047^{*}$ \\
\hline | or || & 21 & $(24.4)$ & 5 & $(13.5)$ & 16 & $(32.7)$ & \\
\hline III or IV & 65 & $(75.6)$ & 32 & $(86.5)$ & 33 & $(67.3)$ & \\
\hline Lauren classification & & & & & & & 0.49 \\
\hline Intestinal type & 31 & $(36.0)$ & 13 & $(35.1)$ & 18 & $(36.7)$ & \\
\hline Diffuse type & 39 & $(45.3)$ & 19 & $(51.4)$ & 20 & $(40.8)$ & \\
\hline Mixed type & 16 & (18.6) & 5 & $(13.5)$ & 11 & $(22.4)$ & \\
\hline Cell differentiation & & & & & & & 0.54 \\
\hline Well-differentiated & 7 & $(8.2)$ & 4 & $(11.1)$ & 3 & $(6.1)$ & \\
\hline Moderately differentiated & 23 & $(27.1)$ & 11 & $(30.6)$ & 12 & $(24.5)$ & \\
\hline Poorly- or un-differentiated & 55 & $(64.7)$ & 21 & $(58.3)$ & 34 & $(69.4)$ & \\
\hline LN metastasis (number) & 4.5 & \pm 5.7 & 5.2 & \pm 7.8 & 3.9 & \pm 3.3 & 0.35 \\
\hline LN harvested (number) & 34.9 & \pm 18.6 & 33.9 & \pm 19.3 & 35.6 & \pm 18.2 & 0.67 \\
\hline Lymphovascular invasion & 56 & $(65.1)$ & 25 & $(67.6)$ & 31 & $(63.3)$ & 0.85 \\
\hline Perineural invasion & 57 & $(66.3)$ & 25 & $(67.6)$ & 32 & $(65.3)$ & 0.99 \\
\hline T stage (AJCC $8^{\text {th }}$ ) & & & & & & & $<0.001^{*}$ \\
\hline 1 & 0 & $(0)$ & 0 & (0) & 0 & (0) & \\
\hline 2 & 19 & $(21.8)$ & 8 & $(21.6)$ & 11 & $(22.4)$ & \\
\hline 3 & 42 & $(48.3)$ & 8 & $(21.6)$ & 33 & $(67.3)$ & \\
\hline 4 & 26 & $(29.9)$ & 21 & $(56.8)$ & 5 & $(10.2)$ & \\
\hline$N$ stage (AJCC $8^{\text {th }}$ ) & & & & & & & 0.48 \\
\hline 0 & 2 & $(2.3)$ & 0 & (0) & 2 & $(4.1)$ & \\
\hline 1 & 33 & $(38.4)$ & 15 & $(40.5)$ & 18 & $(36.7)$ & \\
\hline 2 & 36 & $(41.9)$ & 14 & $(37.8)$ & 22 & $(44.9)$ & \\
\hline 3 & 15 & $(17.4)$ & 8 & $(21.6)$ & 7 & $(14.3)$ & \\
\hline Pathological stage (AJCC $8^{\text {th }}$ ) & & & & & & & 0.23 \\
\hline$\|$ & 33 & $(38.4)$ & 11 & $(29.7)$ & 22 & $(44.9)$ & \\
\hline III & 53 & $(61.6)$ & 26 & $(70.3)$ & 27 & $(55.1)$ & \\
\hline Follow-up (months) & 52.6 & \pm 31.2 & 65.9 & \pm 36.9 & 43.3 & \pm 22.6 & $0.003^{*}$ \\
\hline
\end{tabular}

Data are mean \pm SD or $n(\%)$

UFT tegafur-uracil, S-1 tegafur-gimeracil-oteracil, LN lymph node, AJCC American Joint Cancer Committee

${ }^{*} p$ value $<0.05$ after comparing between the UFT and S- 1 groups 
and T4 lesions $(56.8 \%$ versus $10.2 \% ; p<0.001)$ were observed in the UFT group than in the S-1 group. The follow-up duration was longer in the UFT group (65.9 \pm 36.9 months) than in the S- 1 group $(43.3 \pm 22.6$ months; $p=0.001)$. There were more pathological stage III gastric cancers in the UFT group than in the S-1 group (70.3\% versus $55.1 \%)$ but with no statistical significance $(p=0.23)$.

The 5 -year RFS rate was $56.1 \%$ in the UFT group and $59.6 \%$ in the S-1 group (Fig. 2a), and the 5 -year OS rate was $78.3 \%$ in the UFT group and $73.1 \%$ in the S-1 group (Fig. 2b). The hazard ratio for recurrence (or all-cause mortality) and all-cause mortality in the $\mathrm{S}-1$ group as compared with the UFT group was 1.14 ( $95 \%$ CI, 0.56 to $2.30 ; p$ value $=0.71)$ and $1.41(95 \% \mathrm{CI}, 0.53$ to $3.73 ; p$ value $=0.48)$, respectively. The total number of recurrences was comparable between the UFT and S-1 groups ( $43.2 \%$ versus $38.8 \% ; p=0.68)$, and similarly, there was no statistical difference in sites of the first recurrence (UFT versus S-1), including local recurrence (10.8\% versus $4.1 \% ; p=0.40)$, lymph nodes $(16.2 \%$ versus $8.2 \%$; $p=0.32)$, peritoneal carcinomatosis $(16.2 \%$ versus $18.4 \%$; $p=0.79)$, and hematogenous metastasis $(16.2 \%$ versus $20.4 \% ; p=0.62$ ), between these two groups (Supplementary Table 1). After Borrmann type, $\mathrm{T}$, and $\mathrm{N}$ stages were adjusted, $\mathrm{N}$ stage (N3 versus $\mathrm{N} 1$ ) was the only significant predicting factor for RFS (HR $=2.86,95 \% \mathrm{CI}$ $=1.16$ to $7.03 ; p=0.02)$ while type of adjuvant chemotherapy (S-1 versus UFT) remained insignificant for RFS $(\mathrm{HR}=1.25 ; 95 \% \mathrm{CI}=0.53$ to $2.94 ; p=0.61)$ (Table 2$)$.

Twenty-seven $(72.9 \%)$ patients in the UFT group and $36(73.5 \%)$ patients in the S-1 group completed the adjuvant chemotherapy $(p=0.96)$, and for those completing the adjuvant chemotherapy, $10(37.0 \%)$ patients in the UFT group, and 19 (52.8\%) patients in the S-1 group required either dose reduction or schedule modification ( $p$ $=0.21$ ) (Table 3). The most common reason for chemotherapy discontinuation was disease progression (4 patients in the UFT group versus 10 patients in the S-1 group), followed by adverse event (3 patients in the UFT group versus 3 patients in the S-1 group) (Table 3 ).

The most common all-grade adverse events were weight loss (27.0\%) and constipation (27.0\%), anemia (21.6\%), followed by nausea (18.9\%) in the UFT group, and were abdominal pain (34.7\%), diarrhea (22.4\%), followed by weight loss (20.4\%) in the S-1 group. The most common $\geq$ grade 3 adverse event was nausea (5.4\%) in the UFT group, and anemia (4.1\%) and weight loss $(4.1 \%)$ in the S-1 group. Hand-foot skin reaction was relatively rare and observed only in the mild form $(<$ grade 3 ) in two (5.4\%) patients in the UFT group and three (6.1\%) patients in the S-1 group (Supplementary Table 2).

\section{Discussion}

This study showed the comparable outcomes of 5-year RFS and OS for patients with pathological stage II-IIIB (excluding T1 cases; AJCC 8th) gastric cancer receiving adjuvant UFT or S-1 monotherapy following D2 gastrectomy by the same surgical team. The type of adjuvant chemotherapy, UFT or S-1, remained insignificant for RFS after Borrmann type, $\mathrm{T}$, and $\mathrm{N}$ stages were adjusted. Although the adjuvant chemotherapy completion rate was similar between the UFT and S-1 groups, there were slightly more dose reductions and/or schedule modifications in the S-1 group.

Oral UFT has less been studied and reported as the adjuvant chemotherapy for advanced gastric cancer.
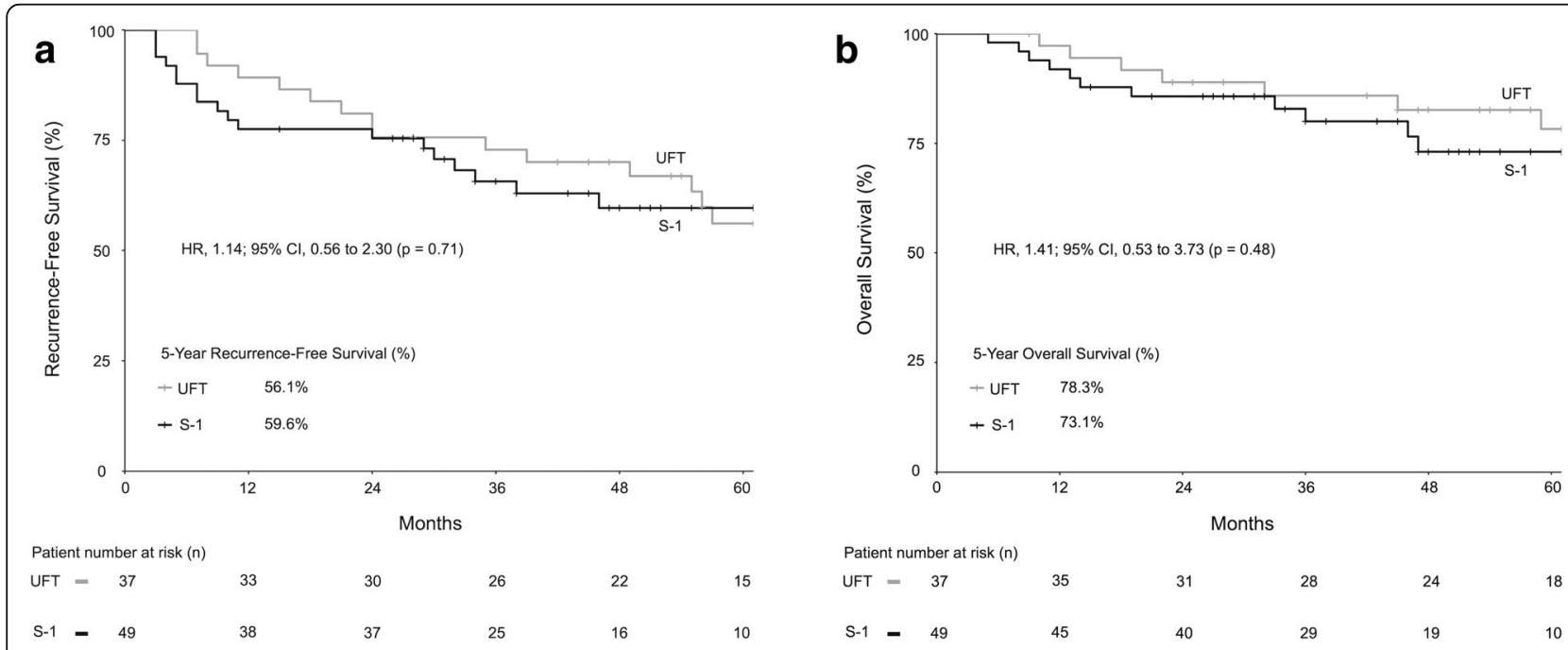

Fig. 2 Kaplan-Meier analysis of 5-year recurrence-free survival (a) and overall survival (b).UFT, tegafur-uracil; S-1, tegafur-gimeracil-oteracil; HR, hazard ratio; $\mathrm{Cl}$, confidence interval 
Table 2 Analysis of important risk factors on recurrence-free survival time using multivariate Cox proportional hazards model

\begin{tabular}{llll}
\hline & HR & $\mathbf{9 5 \% ~ C l}$ & $\boldsymbol{p}$ value \\
\hline Type of chemotherapy (S-1 vs UFT) & 1.25 & $0.53-2.94$ & 0.61 \\
Borrmann type (III/IV vs I/II) & 1.00 & $0.38-2.69$ & 0.99 \\
T stage (T3 vs T2) & 2.99 & $0.96-9.36$ & 0.05 \\
T stage (T4 vs T2) & 2.59 & $0.72-9.29$ & 0.14 \\
N stage (N2 vs N1) & 1.25 & $0.53-2.92$ & 0.61 \\
N stage (N3 vs N1) & 2.86 & $1.16-7.03$ & $0.02^{*}$ \\
\hline
\end{tabular}

HR hazard ratio, Cl confidence interval, UFT tegafur-uracil,

$S-1$ tegafur-gimeracil-oteracil

${ }^{*} p$ value $<0.05$

Nakajima et al. reported an RCT regarding adjuvant treatment with UFT monotherapy for pathological stage T2N1-2 gastric cancer following gastrectomy in 2007 [9], showing that the 5 -year RFS rate was $85 \%$ in the UFT and $68 \%$ in the surgery-only groups $(p=0.005)$, and the 5 -year OS rate was $86 \%$ in the UFT and $73 \%$ in the surgery-only groups $(p=0.017)$, respectively. Although the results were promising, the treatment efficacy for pathological stage $>\mathrm{T} 2 \mathrm{~N} 2$ remained unclear. Later on, the ACTS-GC reported that adjuvant S-1 monotherapy had the 5-year RFS and OS survival benefits for pathological stage II-IIIB (T1 disease excluded; Japanese Classification of Gastric Carcinoma, 2nd English edition) gastric cancer in 2011. The 5-year RFS and OS rates in the ACTS-GC were greater in the S1 group (5-year RFS: 65.4\%; 5-year OS: 71.7\%) than in the surgery-only group (5-year RFS: 53.1\%; 5-year OS: 61.1\%) [7, 8]. Similarly, adjuvant capecitabine (Xeloda ${ }^{\circ}$, another oral fluoropyrimidine, plus oxaliplatin, was also shown effective for pathological stage II-IIIB (AJCC 6th) gastric cancer in the CLASSIC trial in $2012[5,6]$. The 5 -year RFS and OS rates in the CLASSIC trial were greater in the capecitabine-oxaliplatin group (5-year RFS: 68\%; 5-year OS: 78\%) than in the surgery-only group (5-year RFS: 53\%; 5-year OS: 69\%) [5, 6]. The successes of these two large RCTs established the role of oral fluoropyrimidines (S-1 and capecitabine) in the adjuvant setting for advanced gastric cancer; however, UFT seemed to be overlooked thereafter. In 2014, the SAMIT, a two-by-two factorial RCT, aimed initially to evaluate the survival benefit of sequential use of paclitaxel and oral fluoropyrimidines (UFT or S-1) for pathological T4a/4b gastric cancer [15]. The addition of paclitaxel was not associated with significant survival benefits; nevertheless, the results incidentally revealed adjuvant UFT monotherapy was inferior to S-1 monotherapy for patients with pathological $\mathrm{T} 4 \mathrm{a} / 4 \mathrm{~b}$ gastric cancer following D2 gastrectomy (3-year RFS: 53\% versus $58.2 \% ; p<0.001$ ) [15].

The RFS and OS rates of the UFT or S-1 group in our study were inferior to those in the trial conducted by Nakajima et al. (T2N1-2), slightly inferior to those in the ACTS-GC (stage II-IIIB; Japanese classification) and CLASSIC (stage II-IIIB; AJCC 6th) trial, and superior to those in the SAMIT (T4a/b lesions) [5-9, 15]. The differences in survival outcomes between our study and aforementioned clinical trials may result from (1) various patient characteristics and inclusion criteria for adjuvant chemotherapy (including the effects of stage migration between different editions of gastric cancer classification); (2) different regimens, dosages, or durations of adjuvant chemotherapy; (2) retrospective nature of our study. Patients in the UFT/S1 group in this study comprised $56.8 \% / 10.4 \%$ of pathological T4 lesions, $21.6 \% /$ $14.3 \%$ of N3 (AJCC 8th) lesions ( $\geq 7$ metastatic lymph nodes), and $70.3 \% / 55.1 \%$ of stage III (AJCC 8 th) gastric cancer. Patients randomized to the S- 1 adjuvant chemotherapy arm in the ACTS-GC had $2.3 \%$ of pathological T4 lesions, $26.6 \%$ of $\geq 7$ metastatic lymph nodes (equivalent to N3 in the AJCC 8th), and $48 \%$ of stage III (AJCC 6th) gastric cancer, and those in the CLASSIC trial had $1 \%$ of pathological T4 lesions, $31 \%$ of N2 (AJCC 6 th; $\geq 7$ metastatic lymph nodes) lesions (equivalent to N3 in the AJCC 8th), and $51 \%$ of stage III (AJCC 6th) gastric cancer $[5,7]$. It was difficult to compare studies with different patient selection criteria. In this study, although the long-term oncological outcomes (5-year RFS and OS)

Table 3 Completion rates of adjuvant chemotherapy

\begin{tabular}{|c|c|c|c|c|c|}
\hline & UFT & $(n=37)$ & S-1 & $(n=49)$ & $p$ value ${ }^{\#}$ \\
\hline Total number of completions & 27 & (72.9) & 36 & (73.5) & 0.96 \\
\hline Dose reduction or schedule modification & 10 & $(37.0)$ & 19 & $(52.8)$ & 0.21 \\
\hline Total number of discontinuations & 10 & $(27.0)$ & 13 & $(26.5)$ & 0.96 \\
\hline Adverse event & 3 & $(30.0)$ & 3 & $(23.1)$ & 0.10 \\
\hline Disease progression & 4 & $(40.0)$ & 10 & $(76.9)$ & \\
\hline Drop out & 3 & $(30.0)$ & 0 & (0) & \\
\hline
\end{tabular}

Data are number of patients (\%)

UFT tegafur-uracil, $S-1$ tegafur-gimeracil-oteracil

"Comparison of completion rates between the UFT and S-1 groups 
were slightly inferior to those in the ACTS-GC and CLASSIC trial, there were actually more T4 lesions and stage III gastric cancers in our patients.

Comparisons between and the real-world experiences of the different oral fluoropyrimidines remain suboptimal. SAMIT, the only clinical trial evaluating the efficacy of UFT and S-1 for advanced gastric cancer, reported the 3-year RFS benefit for S-1 (HR = 0.81; $p=0.005)$; however, more extended oncological outcomes were still unknown [15]. In this study, the two adjuvant treatment cohorts were formed with the evolution of drug availability and reimbursement policy in the Taiwanese healthcare system, where UFT was available first then followed by S-1 10 years later, and the long-term oncological outcomes (5-year RFS and OS) were comparable between these two groups. Furthermore, the type of adjuvant chemotherapy (S-1 versus UFT) remained insignificant for RFS after Borrmann type, $\mathrm{T}$, and $\mathrm{N}$ stages were adjusted. Compared to the SAMIT's protocol, we adopted the same dosage of UFT but the duration was inevitably extended to 96 weeks. The longer duration (96 weeks versus 48 weeks in the SAMIT) and better chemotherapy complete rate $(72.9 \%$ versus $60 \%$ in the SAMIT) of the UFT treatment may contribute to the survival benefit through the metronomic effect seen in this study $[18,19,21-23]$. Moreover, UFT was proved to have a unique antiangiogenic mechanism through its metabolites, $\gamma$-butyrolactone and $\gamma$-hydroxybutyric acid, in addition to direct cytotoxic effect from its active metabolite, 5-FU; nevertheless, whether these collateral metabolites could exert anti-tumor activity and provide survival benefit requires more investigations [24].

Tolerability and treatment completion rate also play an important role and affect long-term outcomes in adjuvant chemotherapy of advanced gastric cancer. Theoretically, S-1 should reduce gastrointestinal toxicity through the effect of oteracil, but any $\geq$ grade 3 gastrointestinal toxicities, including nausea, diarrhea, constipation, and abdominal pain, were similar between patients receiving UFT and S-1 treatments in this study (Supplementary Table 2) [13]. In line with our findings, the toxicity profiles of any $\geq$ grade 3 gastrointestinal toxicities were also similar between these two regimens in the SAMIT [15]. The optimal molar ratio of tegafur/gimeracil/oteracil and toxicity profile of S-1 were based on several animal studies but has less been compared with that of UFT $[25,26]$. These discrepancies observed in the SAMIT and this study may also imply the necessity of re-evaluating the UFT in clinical use. Apart from the prolonged-course UFT treatment, the S-1 treatment was selectively extended to 12 courses ( 72 weeks) if dose reduction was required for adverse events. The rationale behind this strategy was also the concept of metronomic effect and to maintain the same total dosage of S-1 chemotherapy for dose-reduced
S-1 group [21-23]. The strategy did not compromise the chemotherapy completion rates in this study, which were $72.9 \%$ in the UFT group and $73.5 \%$ in the S-1 group, compared to $65.8 \%$ in the ACTS-GC (S1 arm), $67 \%$ in the CLASSIC trial (capecitabine-oxaliplatin arm), and $60 \%$ in the SAMIT (UFT arm), and 62\% in the SAMIT (S1 arm) $[5,7,15]$. Recently, emerging evidences also indicated that a longer duration of S-1 adjuvant chemotherapy had survival benefit for pathological stage II, III, and metastatic gastric cancer [27-29]; however, more prospective clinical trials are needed to confirm these results from retrospective data and further define a standard treatment duration with a corresponding dose.

Although this cohort study included limited number of patients, we presented the unique experiences and longterm oncological outcomes of using UFT or S-1 adjuvant monotherapy for advanced gastric cancer in Taiwan. The recent pandemic of COVID-19 reminds us that pharmaceutical supply chain may be unstable and re-evaluation of the pre-existing chemotherapeutic agents is necessary. Despite the fact that S-1 and capecitabine are the two main fluoropyrimidines used for advanced gastric cancer as adjuvant chemotherapy in this era, our study implies that UFT may also be a feasible alternative. Nevertheless, our study had some limitations. First, the patient numbers were much smaller than those in the RCTs, because we only included patients from the same surgical team to ensure the surgical quality, consistency of the D2 gastrectomy, and the same strategy for adjuvant chemotherapy. Second, the retrospective nature of the study and lack of additional active control groups using other adjuvant regimens, such as CAPOX (capecitabine + oxaliplatin) or P-HDFL (cisplatin + infusional high-dose 5-FU), also limited the comparisons and implications.

\section{Conclusions}

Adjuvant UFT and S-1 monotherapy had comparable long-term outcomes for pathological stage II-IIIB gastric cancer following D2 gastrectomy based on the realworld data from our small cohort study.

\section{Abbreviations \\ RCT: Randomized controlled trial; DPD DIF: Dihydropyrimidine dehydrogenase inhibitory fluoropyrimidine; 5-FU: 5-Flurouracil; UFT: Tegafur- uracil; S-1: Tegafur-gimeracil-oteracil; ACTS-GC: Adjuvant chemotherapy trial of TS-1 for gastric cancer; SAMIT: Stomach cancer Adjuvant Multi-Institutional group Trial; COVID-19: Coronavirus disease; AJCC: American Joint Committee on Cancer; RFS: Recurrence-free survival; OS: Overall survival; HR: Hazard ratio; $\mathrm{Cl}$ : Confidence interval}

\section{Supplementary Information}

The online version contains supplementary material available at https://doi. org/10.1186/s12957-021-02233-2.

Additional file 1: Supplementary Table 1. Site of the first recurrence. Supplementary Table 2. Adverse events 


\section{Acknowledgements}

The long-term follow-up data were provided by Cancer Registry, Cancer Administration, and Coordination Center, National Taiwan University Hospital. This study was granted by the Liver Disease Prevention \& Treatment Research Foundation (Taiwan) and by the Ministry of Science and Technology (Taiwan) (MOST 103-2320-B-002-005)

\section{Authors' contributions}

Conception and design of study: H.-H.Y., C.-N.C., C.-C.Y., I.-R.L. Acquisition of data: H.-H.Y., C.-N.C., C.-C.Y., I.-R.L. Analysis and/or interpretation of data: H.H.Y., I.-R.L. Drafting the manuscript: H.-H.Y., I.-R.L. Revising the manuscript critically for important intellectual content: H.-H.Y., I.-R.L. All authors have read and approved the final version of the manuscript.

\section{Funding}

The authors declare that they had no funding to prepare this report.

\section{Availability of data and materials}

The datasets generated and/or analyzed during the current study are not publicly available but are available from the corresponding author on reasonable request.

\section{Declarations}

\section{Ethics approval and consent to participate}

All procedures followed were in accordance with the ethical standards of the institutional and national committees governing human experimentation and in compliance with the Helsinki Declaration of 1964 and later versions. The conduction of this retrospective study was approved by the ethics committee of the National Taiwan University Hospital and eligible for a waiver of informed consent (NTUH-REC No.: 202101037RINB).

\section{Consent for publication}

Not applicable.

\section{Competing interests}

The authors declare that they have no competing interests.

\begin{abstract}
Author details
'Department of Surgery, National Taiwan University Biomedical Park Hospital, Hsinchu County, Taiwan. ${ }^{2}$ Department of Surgery, National Taiwan University Hospital, No. 7 Chung-Shan South Rd, Zhongzheng District, Taipei 10002, Taiwan. ${ }^{3}$ Department of Medical Education, National Taiwan University Hospital, Taipei, Taiwan. ${ }^{4}$ Graduate Institute of Anatomy and Cell Biology, College of Medicine, National Taiwan University, Taipei, Taiwan.
\end{abstract}

\section{Received: 13 January 2021 Accepted: 6 April 2021}

\section{Published online: 17 April 2021}

\section{References}

1. Bray F, Ferlay J, Soerjomataram I, Siegel RL, Torre LA, Jemal A. Global cancer statistics 2018: GLOBOCAN estimates of incidence and mortality worldwide for 36 cancers in 185 countries. CA Cancer J Clin. 2018;68(6):394-424. https://doi.org/10.3322/caac.21492.

2. Songun I, Putter H, Kranenbarg EM, Sasako M, van de Velde CJ. Surgical treatment of gastric cancer: 15-year follow-up results of the randomised nationwide Dutch D1D2 trial. Lancet Oncol. 2010;11(5):439-49. https://doi. org/10.1016/S1470-2045(10)70070-X.

3. Smyth EC, Verheij M, Allum W, Cunningham D, Cervantes A, Arnold D, et al. Gastric cancer: ESMO clinical practice guidelines for diagnosis, treatment and follow-up. Ann Oncol. 2016;27(suppl 5):v38-49. https://doi.org/10.1 093/annonc/mdw350.

4. Ajani JA, D'Amico TA, Almhanna K, Bentrem DJ, Chao J, Das P, et al. Gastric cancer, version 3.2016, NCCN clinical practice guidelines in oncology. J Natl Compr Canc Netw. 2016;14(10):1286-312. https://doi.org/10.6004/jnccn.201 6.0137.

5. Bang YJ, Kim YW, Yang HK, Chung HC, Park YK, Lee KH, et al. Adjuvant capecitabine and oxaliplatin for gastric cancer after D2 gastrectomy (CLAS SIC): a phase 3 open-label, randomised controlled trial. Lancet. 2012; 379(9813):315-21. https://doi.org/10.1016/50140-6736(11)61873-4.
6. Noh SH, Park SR, Yang HK, Chung HC, Chung IJ, Kim SW, et al. Adjuvant capecitabine plus oxaliplatin for gastric cancer after D2 gastrectomy (CLAS SIC): 5-year follow-up of an open-label, randomised phase 3 trial. Lancet Oncol. 2014;15(12):1389-96. https://doi.org/10.1016/S1470-2045(14)70473-5.

7. Sakuramoto S, Sasako M, Yamaguchi T, Kinoshita T, Fujii M, Nashimoto A, et al. Adjuvant chemotherapy for gastric cancer with S-1, an oral fluoropyrimidine. N Engl J Med. 2007;357(18):1810-20. https://doi.org/10.1 056/NEJMoa072252.

8. Sasako M, Sakuramoto S, Katai H, Kinoshita T, Furukawa H, Yamaguchi T, et al. Five-year outcomes of a randomized phase III trial comparing adjuvant chemotherapy with S-1 versus surgery alone in stage II or III gastric cancer. J Clin Oncol. 2011;29(33):4387-93. https://doi.org/10.1200/JCO.2011.36.5908.

9. Nakajima T, Kinoshita T, Nashimoto A, Sairenji M, Yamaguchi T, Sakamoto J, et al. Randomized controlled trial of adjuvant uracil-tegafur versus surgery alone for serosa-negative, locally advanced gastric cancer. Br J Surg. 2007; 94(12):1468-76. https://doi.org/10.1002/bjs.5996.

10. Aykan NF, Idelevich E. The role of UFT in advanced gastric cancer. Ann Oncol. 2008;19(6):1045-52. https://doi.org/10.1093/annonc/mdn024.

11. Japanese Gastric Cancer A. Japanese gastric cancer treatment guidelines 2018 (5th edition). Gastric Cancer. 2021;24:1-21.

12. Guideline Committee of the Korean Gastric Cancer Association DWG, Review P. Korean Practice Guideline for Gastric Cancer 2018: an evidencebased, multi-disciplinary approach. J Gastric Cancer. 2019;19:1-48.

13. Maehara Y. S-1 in gastric cancer: a comprehensive review. Gastric Cancer. 2003;6(Suppl 1):2-8. https://doi.org/10.1007/s10120-003-0232-9.

14. Kinoshita T, Nashimoto A, Yamamura Y, Okamura T, Sasako M, Sakamoto J, et al. Feasibility study of adjuvant chemotherapy with S-1 (TS-1; tegafur, gimeracil, oteracil potassium) for gastric cancer. Gastric Cancer. 2004;7(2): 104-9. https://doi.org/10.1007/s10120-004-0278-3.

15. Tsuburaya A, Yoshida K, Kobayashi M, Yoshino S, Takahashi M, Takiguchi N, et al. Sequential paclitaxel followed by tegafur and uracil (UFT) or S-1 versus UFT or S-1 monotherapy as adjuvant chemotherapy for T4a/b gastric cancer (SAMIT): a phase 3 factorial randomised controlled trial. Lancet Oncol. 2014; 15(8):886-93. https://doi.org/10.1016/S1470-2045(14)70025-7.

16. Japanese Gastric Cancer A. Japanese classification of gastric carcinoma: 3rd English edition. Gastric Cancer. 2011;14:101-12.

17. Yeh CC, Yen HH, Lai IR. Laparoscopic distal gastrectomy for clinical stage I gastric adenocarcinoma: techniques evolution and oncological outcomes of the first 100 cases. J Formos Med Assoc. 2019;1 18(1):179-85. https://doi. org/10.1016/j.jfma.2018.03.010

18. Sanford M. S-1 (Teysuno(R)): a review of its use in advanced gastric cancer in non-Asian populations. Drugs. 2013;73(8):845-55. https://doi.org/10.1007/ s40265-013-0062-y.

19. Hoff PM, Saad ED, Ajani JA, Lassere Y, Wenske C, Medgyesy D, et al. Phase I study with pharmacokinetics of S-1 on an oral daily schedule for 28 days in patients with solid tumors. Clin Cancer Res. 2003;9(1):134-42.

20. Hu B, El Hajj N, Sittler S, Lammert N, Barnes R, Meloni-Ehrig A. Gastric cancer: classification, histology and application of molecular pathology. J Gastrointest Oncol. 2012;3:251-61.

21. Hanahan $D$, Bergers $G$, Bergsland E. Less is more, regularly: metronomic dosing of cytotoxic drugs can target tumor angiogenesis in mice. J Clin Invest. 2000;105(8):1045-7. https://doi.org/10.1172/JCl9872.

22. Simsek C, Esin E, Yalcin S. Metronomic chemotherapy: a systematic review of the literature and clinical experience. J Oncol. 2019;2019:5483791.

23. Iwasa S, Yamada Y, Fukagawa T, Eguchi Nakajima T, Kato K, Hamaguchi T, et al. Management of adjuvant S-1 therapy after curative resection of gastric cancer: dose reduction and treatment schedule modification. Gastric Cancer. 2011;14(1):28-34. https://doi.org/10.1007/s10120-011-0003-y.

24. Yonekura K, Basaki Y, Chikahisa L, Okabe S, Hashimoto A, Miyadera K, et al. UFT and its metabolites inhibit the angiogenesis induced by murine renal cell carcinoma, as determined by a dorsal air sac assay in mice. Clin Cancer Res. 1999;5(8):2185-91.

25. Shirasaka T, Shimamato $Y$, Ohshimo $H$, Yamaguchi $M$, Kato $T$, Yonekura $K$, et al. Development of a novel form of an oral 5-fluorouracil derivative (S-1) directed to the potentiation of the tumor selective cytotoxicity of 5fluorouracil by two biochemical modulators. Anticancer Drugs. 1996;7(5): 548-57. https://doi.org/10.1097/00001813-199607000-00010.

26. Kato T, Shimamoto Y, Uchida J, Ohshimo H, Abe M, Shirasaka T, et al. Possible regulation of 5-fluorouracil-induced neuro- and oral toxicities by two biochemical modulators consisting of S-1, a new oral formulation of 5fluorouracil. Anticancer Res. 2001;21(3B):1705-12. 
27. Fujitani K, Kurokawa Y, Takeno A, Endoh S, Ohmori T, Fujita J, et al. Time to initiation or duration of S-1 adjuvant chemotherapy; which really impacts on survival in stage II and III gastric cancer? Gastric Cancer. 2018;21(3):44652. https://doi.org/10.1007/s10120-017-0767-9.

28. Yoshikawa T, Terashima M, Mizusawa J, Nunobe S, Nishida Y, Yamada T, et al. Four courses versus eight courses of adjuvant S-1 for patients with stage II gastric cancer (JCOG1104 [OPAS-1]): an open-label, phase 3, noninferiority, randomised trial. Lancet Gastroenterol Hepatol. 2019;4(3):208-16. https://doi.org/10.1016/S2468-1253(18)30383-2

29. Haag GM, Stocker G, Quidde J, Jaeger D, Lordick F. Randomized controlled trial of S-1 maintenance therapy in metastatic esophagogastric cancer - the multinational MATEO study. BMC Cancer. 2017;17(1):509. https://doi.org/1 0.1186/s12885-017-3497-9.

\section{Publisher's Note}

Springer Nature remains neutral with regard to jurisdictional claims in published maps and institutional affiliations.

Ready to submit your research? Choose BMC and benefit from:

- fast, convenient online submission

- thorough peer review by experienced researchers in your field

- rapid publication on acceptance

- support for research data, including large and complex data types

- gold Open Access which fosters wider collaboration and increased citations

- maximum visibility for your research: over $100 \mathrm{M}$ website views per year

At BMC, research is always in progress.

Learn more biomedcentral.com/submissions 